\title{
Consideraciones de las propiedades mecánicas y térmicas para la elaboración de un ducto de riego a partir de materiales poliméricos postconsumo
}

\author{
Considerations of mechanical and thermal \\ properties for the production of an irrigation \\ pipeline from post-consumer polymer materials
}

Mary Luz Barrios-Hernández'

Fecha de recepción: 22 de octubre del 2014

Fecha de aprobación: 04 de marzo del 2014

Barrios-Hernández, M. Consideraciones de las propiedades mecánicas y térmicas para la elaboración de un ducto de riego a partir de materiales poliméricos postconsumo. Tecnología en Marcha. Vol. 27, № 3, Julio-Setiembre 20 I4. Pág 


\section{Palabras clave}

Mezclas de polímeros; poliducto de riego; polietileno de alta densidad; polietileno de baja densidad; polipropileno.

\section{Resumen}

En este artículo se presentan los resultados obtenidos al desarrollar tres nuevas formulaciones a partir de materiales poliméricos generados en el desecho doméstico, con el fin de determinar el mejor material para fabricar poliducto de riego en Costa Rica, utilizando poliducto comercial como referencia. Se recuperó material polimérico postconsumo, específicamente polietilenos y polipropilenos, con la ayuda de actores de programas de recuperación de desechos del Tecnológico de Costa Rica. El material requirió de procesos como limpieza, clasificación, molienda y la extrusión de láminas. Las muestras se prepararon con diferentes proporciones de los polímeros utilizados. Un primer especimen fue de polietileno de baja densidad, otra mezcla fue de $75 \%$ de polietileno de baja densidad y $25 \%$ de polietileno de alta densidad, y una última mezcla de $75 \%$ de polietileno de alta densidad y $25 \%$ de polipropileno. A estas muestras se les determinaron propiedades mecánicas y térmicas.

Como principal resultado se obtuvo que la formulación que mejoró las propiedades estudiadas del poliducto fue la mezcla de diferentes productos de Polietileno de Baja Densidad postconsumo, para un total de 10 repeticiones con 95\% de confianza en la prueba de Kolmogorov-Smirnov, concluyendo que este producto supera las propiedades de resistencia en elongación y flexión y que necesita la optimización de las características para resistencia al impacto modo IZOD. Además, la prueba de calorimetría diferencial de barrido demostró la ausencia de agentes externos en la muestras y el análisis termogravimétrico reveló cambios de temperatura de descomposición semejantes al ducto y mayores a las otras mezclas.

\section{Key words}

Polymer blends; irrigation pipeline; high density polyethylene; low density polyethylene; polypropylene.

\begin{abstract}
It aims to show the results to develop three new formulations of polymeric materials from household waste generated, in order to determine the best material to make irrigation pipeline in Costa Rica, using commercial pipeline reference. Polymeric material was recovered polyethylene and polypropylene post-consumer, with the help of ITCR waste recovery programs. The material required for processes such as cleaning, sorting, grinding and extrusion. The samples were prepared with different proportions of the polymers used. The first specimen was LDPE; another mix was $75 \%$ of LDPE and $25 \%$ HDPE and a final mixture of $75 \%$ of HDPE and $25 \%$ of PP. These samples were analyzed for mechanical and thermal properties.
\end{abstract}

As a main result we found that the improved formulation of the pipeline properties studied was the mixture of different post-consumer LDPE products, for a total of ten repetitions with $95 \%$ confidence in the Kolmogorov- Smirnov test, concluding that this product exceeds elongation resistance properties in bending and optimization needed characteristics for IZOD impact strength mode. Testing differential scanning calorimetry showed no external agents in samples, and thermogravimetric analysis showed the decomposition temperature change similar to the duct and higher than the other blends. 


\section{Introducción}

En la búsqueda de nuevas opciones para el tratamiento de los plásticos postconsumo se da la posibilidad de reutilizar estos materiales y prolongar su vida útil, por medio de la generación de nuevas materias primas a partir de la producción de mezclas de polímeros. Esto permite producir beneficios económicos, ambientales y sociales e implementar un manejo más armonioso de los plásticos de gran consumo. Según el Programa Estado de la Nación 20I I, se estima que en Costa Rica se producen unas 2700 toneladas diarias de basura proveniente de los hogares; de estas, los desechos plásticos representan el $9 \%$ del peso total de la basura doméstica. Entre los desechos plásticos más comunes que se pueden recolectar y reciclar están el polietilentereftalato, el polietileno de alta densidad (PEAD), el policloruro vinílico, el polietileno de baja densidad (PEBD), el polipropileno (PP) y el poliestireno, entre otros (Osswald, 20 I I).

Un polímero viene a ser una molécula que consta de (poli-) muchas partes (- mer) unidos entre sí por enlaces químicos covalentes. Las partes individuales, o segmentos de monómero, de un polímero pueden ser todas del mismo elemento (Kulshreshtha y Vasile, 2002). El polietileno y el polipropileno, materiales que fueron estudiados en esta investigación, son conocidos como los dos primeros miembros de la cadena de los polímeros y están basados en la estructuras del etileno; según su clasificación química, pertenecen a la familia de las poliolefinas. Son semejantes en apariencia, sin embargo, las diferencias radican en las propiedades físicas y termoestables (Haper, 1975).

Estos polímeros son altamente utilizados en la agricultura. Los agricultores obtienen importantes rendimientos gracias al aporte de estos materiales poliméricos, entre ellos se encuentra un producto para riego denominado poliducto. Se considera un poliducto comercial que los agricultores someten a altas tensiones por largos estiramientos en condiciones ambientales muy variables.

Un método de comparación y caracterización de los polímeros es partir de sus propiedades mecánicas y térmicas, las cuales han sido muy estudiadas para polímeros o resinas con alto grado de pureza. Entre las propiedades mecánicas se ha estudiado la resistencia en elongación, para determinar la deformación que muestra el cambio en la forma que experimenta el ducto bajo tensión; es decir, que la muestra se deforma por estiramiento, volviéndose más larga y se mide como elongación elástica (Odian, 1991). Según Harper (1975), la resistencia en flexión al 5\% de deformación es otra propiedad mecánica utilizada como una medida de rigidez requerida para doblar la probeta de ensayo en una cantidad determinada y se utiliza cuando la barra de ensayo se dobla demasiado sin romperse. También se ha estudiado la resistencia al impacto por medio del método IZOD, que representa la resistencia o tenacidad de un material rígido a la repentina aplicación de una carga mecánica.

Respecto a los parámetros térmicos estudiados, la calorimetría diferencial de barrido es una técnica empleada para analizar las transiciones térmicas de un polímero, que son cambios que tienen lugar cuando el polímero se calienta; según Carraher y Seymour (1995), el análisis de TGA mide la cantidad y la velocidad del cambio de peso de una muestra en función de la temperatura y/o del tiempo en una atmósfera controlada. De manera general, permite realizar medidas para determinar la composición de los materiales y predecir su estabilidad a temperaturas de hasta $1000^{\circ} \mathrm{C}$.

Respecto a las mezclas, Parma (20 I I) indica que las mezclas de polímeros usualmente resultan en un sistema inmiscible, dependiendo del grosor, la facilidad de alterar la morfología y la pobre adhesión entre las fases. En particular, se ven afectadas las propiedades mecánicas como la resistencia al impacto, la resistencia en elongación y en flexión, siendo la estructura de las mezclas inestable y hasta irreproducible.

Kulshreshtha y Vasile (2002) han estudiado diferentes mezclas de polietilenos, incluyendo PEAD, PEBD y PP. En la mezcla PEAD/PEBD, el PEAD facilita la cristalización de ramificaciones largas en el PEBD; además, tiene mejoras en la resistencia en elongación, el impacto al péndulo y pequeños cambios en la elasticidad. La mezcla de PEBD/PEAD, procesada en presencia de peróxido de dicumilo (DCP), tiene mejores propiedades mecánicas y al agregar pequeñas cantidades de PP se puede modificar la viscosidad de esta mezcla. Al mezclar PEBD/PP se obtienen cambios mecánicos como mejoras en la resistencia al impacto. Además, Utracki (2002) ha demostrado que las mezclas de diferentes tipos de 
polietileno mejoran las propiedades mecánicas y de procesabilidad del material.

El presente estudio examina el potencial uso de mezclas de polímeros, como el polietileno de alta y baja densidad y el polipropileno postconsumo, como materia prima para la elaboración de poliducto para riego; se caracterizan las propiedades mecánicas y térmicas de los compuestos y se aportan resultados al campo de la investigación científica en el área de los compuestos de plásticos reciclados.

\section{Materiales y métodos}

\section{Preparación de las mezclas}

El material de análisis se obtuvo a través de los programas Ambientados-JASEC y CCTM, empresas recolectoras de material mediante campañas de reciclaje. El acoplamiento del material y la producción de mezclas conllevaron procesos de selección de material, limpieza y molienda para su extrusión. Las botellas se limpiaron de impurezas con agua y se les eliminaron las etiquetas de papel.

El proceso de molienda se llevó a cabo en un molino en el cual se disminuyó la materia prima hasta un tamaño de aproximadamente $4 \mathrm{~cm}^{2}$. En el moldeo por extrusión se utilizó un transportador de tornillo helicoidal. Las mezclas de polímeros fueron homogeneizadas y luego transportadas desde la tolva, a través de la cámara de calentamiento, hasta la boquilla del molde. Las muestras de los dos polímeros reciclados se adicionaron a la tolva, en donde el material dosificado pasó al husillo, que giró concéntricamente en un cilindro a temperaturas controladas al punto de maleabilidad y de fusión del polímero de mayor y menor temperatura respectivamente, luego fue arrastrado por medio de presión y finalmente forzado a pasar a través de un dado para obtener el perfil laminar preestablecido. Se realizaron tres muestras con diferentes composiciones poliméricas (cuadro I).

Se utilizó un ejemplar del poliducto para riego distribuido en Costa Rica como material de referencia para la comparación de las propiedades mecánicas y térmicas de las mezclas y se ajustaron a las características del análisis de las probetas solicitadas por el laboratorio.

Para el análisis estadístico de los datos se aplicó la opción de Kolmogorov-Smirnov, con un nivel de significancia del 5\% para dos medias independientes, comparando las medianas entre cada tratamiento. Los resultados fueron procesados por medio de tablas y gráficos y todos los análisis se realizaron utilizando el software estadístico SPSS Statistics 21.0.

\section{Análisis mecánicos}

Los ensayos mecánicos que se aplicaron fueron resistencia a la tensión, elongación, resistencia a la flexión e impacto al péndulo módulo IZOD. Todas las probetas se acondicionaron a $23 \pm 2^{\circ} \mathrm{C}$ y $50 \pm$ $5 \%$ de humedad relativa durante 48 horas previas al análisis.

\section{Ensayo de tensión}

Los análisis de laboratorio se realizaron en el laboratorio de Polímeros de la Universidad Nacional. Para comprobar la resistencia a la tensión y la elongación, se probaron alrededor de 10 especímenes del tipo IV para cada mezcla y el material de referencia, de acuerdo con la norma ASTM D-638M y D-882. El aparato utilizado fue un analizador mecánico universal INSTRON 3365, con una celda de 5 kN. Se empleó una velocidad de aplicación del esfuerzo de $5 \mathrm{~mm}$ por minuto. La apertura inicial entre las prensas de la máquina fue de $100 \mathrm{~mm}$.

Cuadro I. Composición de las diferentes muestras de polímeros.

\begin{tabular}{|c|c|c|c|c|}
\hline \multirow{2}{*}{ Número de muestra } & Nombre de la mezcla & $\begin{array}{c}\text { PEAD } \\
\%\end{array}$ & $\begin{array}{c}\text { PEBD } \\
\%\end{array}$ & $\begin{array}{c}\text { PP } \\
\%\end{array}$ \\
\hline 1 & PEBD-postconsumo & - & 100 & - \\
2 & PEAD-PEBD & 75 & 25 & - \\
3 & PEAD-PP & 75 & - & 25 \\
\hline
\end{tabular}


Ensayo de impacto

Se determinó el promedio de resistencia al impacto tanto en $\mathrm{J} / \mathrm{m}$ siguiendo el procedimiento ASTM D-256, método A, correspondiente al ensayo IZOD, a las muestras en forma de vigueta $(72 \times 12) \mathrm{mm}$, con una muesca según las indicaciones del método. Se utilizó un péndulo con una capacidad 2,7 J y una longitud de $42 \mathrm{~cm}$.

\section{Ensayo de flexión}

Se siguió el método ASTM D-790 para el análisis de tensión en flexión, probando alrededor de 10 especímenes de perfil rectangular para cada muestra con dimensiones promedio (en $\mathrm{mm}$ ) de $80 \times 12 \times 4$. El aparato utilizado fue un analizador mecánico universal INSTRON 3365, con una celda de 5 kN. Se empleó una configuración de flexión en tres puntos, con una distancia de soportes de $50 \mathrm{~mm}$ y una velocidad de aplicación del esfuerzo en el punto medio de la probeta de 1,3 mm por minuto.

\section{Análisis térmicos}

A todas las muestras se les aplicó análisis de calorimetría diferencial de barrido (DSC), siguiendo el método ASTM 34 I7. La muestra se colocó en una cápsula de aluminio sellada. Se utilizó un ámbito de temperatura de análisis de $50-140{ }^{\circ} \mathrm{C}$, en atmósfera de nitrógeno con un flujo de $20 \mathrm{~mL}$ por minuto y un programa de temperatura de $10^{\circ} \mathrm{C}$ por minuto. Se hizo una primera corrida, luego el material se enfrió y subsecuentemente se hizo una segunda corrida, ambas con el mismo rango de temperatura y la misma velocidad de calentamiento.

El análisis termogravimétrico (TGA) utilizó un analizador termogravimétrico con una rampa de calentamiento de $20^{\circ} \mathrm{C} / \mathrm{min}$ desde 50 hasta $600{ }^{\circ} \mathrm{C}$ y bajo un flujo de nitrógeno en la balanza de $40 \mathrm{~mL} / \mathrm{min}$ y de $60 \mathrm{~mL} / \mathrm{min}$ en la muestra. Se obtuvieron gráficas de pérdida de peso y de variación del peso (primera derivada) versus porcentaje de residuo presente en cada muestra.

La elaboración de las mezclas contempló la determinación de diferentes composiciones poliméricas para cada formulación efectuada. Se utilizó un diseño experimental completamente aleatorio para determinar diferencias significativas entre cada tratamiento, obteniendo la variable respuesta de cada análisis aplicado.

\section{Resultados y discusión}

La prueba de Kolmogorov-Smirnov mostró que el valor de probabilidad "p" fue menor que 0,05 para el modelo, lo cual indica que existieron diferencias significativas entre las mezclas para los tres análisis mecánicos realizados, por lo que se distribuyeron los tratamientos respecto a las medianas poblacionales. En la figura I se puede observar la distribución de los promedios por muestra ante la tensión-estiramiento ejercida a cada una de las 10 probetas estudiadas, comparadas con el comportamiento de los datos promedio del poliducto comercial utilizado como referencia. En cada mezcla se estudió la relación de la tensión respecto a la elongación, resultado de

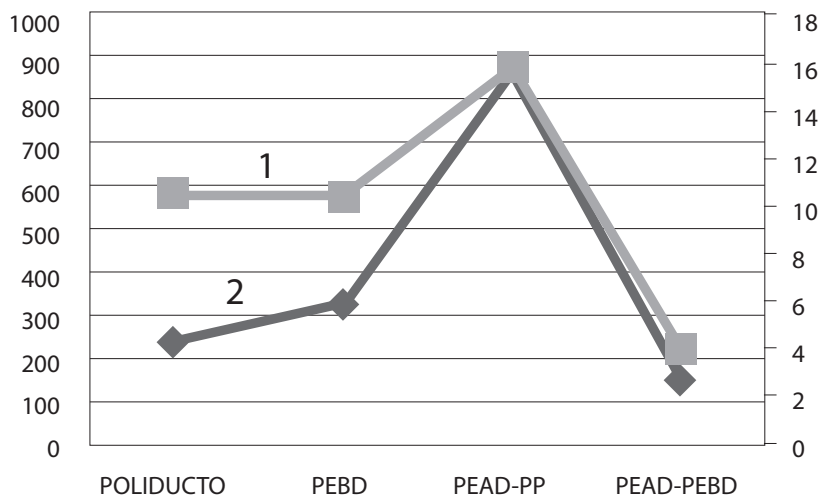

1- Módulo elástico (MPa)

2- Resistencia máxima (MPa)

Figura I. Promedio del módulo elástico y la resistencia máxima a partir de la prueba de resistencia a la elongación para cuatro muestras diferentes. Fuente: POLIUNA 2012. 
graficar una curva de tensión-estiramiento (Odian, 1991). La altura de la curva cuando la muestra se rompió representó la resistencia máxima y la pendiente representó el módulo elástico.

En las mezclas PEBD postconsumo y PEAD-PEBD las pendientes fueron suaves, por lo que se puede decir que las muestras poseen bajos módulos tensiles, por lo tanto se deformaron con facilidad; sin embargo, la mezcla PEAD-PP mostró fuertes rompimientos y caídas de pendiente al punto de su rompimiento, mostrando una baja elongación. La caracterización del poliducto comercial resultó con parámetros mecánicos acordes al polímero PEBD igualmente con pendientes suaves y un muy alto porcentaje de deformación a la ruptura, valor que no suplió ninguna de las mezclas en estudio.

La mezcla de PEBD postconsumo y de PEAD-PP mejoró las propiedades tensiles del material de referencia, contrariamente a la mezcla PEAD-PEBD, que no suple los parámetros propuestos. Utracki (2002) ha demostrado que las mezclas de diferentes tipos de PE mejoran las propiedades mecánicas y de procesabilidad del material, situación que se da para la formulación de PEBD reciclado, sin embargo, esta probabilidad no se cumplió en la composición de dos tipos de polietileno, PEAD-PEBD, resultado que se atribuye a la heterogeneidad de la mezcla que llevó a la formación de grandes burbujas y la apariencia de piel de naranja; cumpliéndose el razonamiento de Parma (20 I I) sobre la inmicibilidad de las fases en las mezclas de polímeros. Las mezclas PEAD-PEBD y PEAD-PP quedan por debajo de las propiedades mecánicas de elongación y flexión requeridas para ser consideradas como materiales aptos para la fabricación de ductos.
En el cuadro 2 se muestran los resultados de la prueba de resistencia en flexión y su módulo elástico. Los materiales pudieron sobrellevar grandes deformaciones, pues a pesar de que se les aplicaron altas cargas no se rompieron. Según Mark (1999), la deformación elástica es reversible y desaparece después de eliminar el estrés, razón por la cual es necesario reportar el nivel de estrés para una deformación al 5\%.

Respecto al análisis de resistencia al impacto al péndulo modo IZOD, Kulshreshtha y Vasile (2002) dicen que en la mezcla PEAD-PEBD, el PEAD facilita la cristalización de ramificaciones largas en el PEBD y que además tiene mejoras en el impacto al péndulo, razonamiento que se cumple solo en el $50 \%$ de las muestras estudiadas de esta mezcla, además de una quebradura parcial en el otro $50 \%$.

En orden de mejora de las propiedades de resistencia al impacto al péndulo modo IZOD del poliducto, la muestra PEBD postconsumo tuvo quebraduras tipo bisagra, donde una parte queda unida a la otra por una pequeña porción de material en el I00\% de los especímenes estudiados. La mezcla de PEADPP-aditivo tuvo un $37,5 \%$ de quebradura total en los especímenes y el resto se quebró tipo bisagra. El material del poliducto comercial no tuvo quebraduras, demostrando una alta capacidad de absorber energía cuando se somete a impactos. A nivel cuantitativo, las muestras estudiadas son de menor calidad y no cumplen con las especificaciones necesarias del poliducto, lo que permite concluir que un ducto de estos materiales no soportaría fuertes dobleces ni enrollamientos, pues se quebrarían con suma facilidad.

Cuadro 2. Determinación de la resistencia en flexión de las muestras en estudio.

\begin{tabular}{|c|c|c|}
\hline \multirow{2}{*}{ MUESTRA } & Modulo elástico & Tensión en flexión al 5\% de deformación \\
\cline { 2 - 3 } & $(\mathrm{MPa})$ & $(\mathrm{MPa})$ \\
\hline POLIDUCTO & $229 \pm 28$ & $8,3 \pm 0,8$ \\
\hline PEBD postconsumo & $396 \pm 17$ & $13,0 \pm 0,4$ \\
\hline PEAD-PP & $784 \pm 103$ & $26,1 \pm 2,8$ \\
\hline PEAD-PEBD & $120 \pm 26$ & $4,2 \pm 0,8$ \\
\hline
\end{tabular}

Fuente: POLIUNA 2012. 
En la figura 2, para el análisis diferencial de barrido, se trazó una curva de flujo de calor a medida que la temperatura se iba incrementando. Sobre el eje " $x$ " se gráfica la temperatura, sobre el eje " $y$ " la diferencia de producción de calor entre dos calefactores, a una temperatura dada. Se pudo determinar la temperatura de fusión, que es una transición que se da en los polímeros cristalinos y ocurre cuando las cadenas poliméricas abandonan sus estructuras cristalinas y se transforman en un líquido desordenado (Rupper, 2002).

Al comparar las temperaturas de fusión de todas las muestras, se reconoce el comportamiento individual de cada uno de los componentes de las mezclas, razón por la cual en el cuadro 3 se muestran dos temperaturas, una del componente que se funde primero y otra del polímero que se funde a una temperatura más alta.

Por ejemplo, la mezcla PEAD-PP reporta solo una temperatura de fusión, pues según POLIUNA (2012) el analizador reconoció un pico de temperatura de $131,8^{\circ} \mathrm{C}$, siendo este el punto de fusión del componente $\mathrm{PEAD}$, que reporta una temperatura de fusión entre $127-135^{\circ} \mathrm{C}$. El PP puro tiene una temperatura de fusión aceptada de $161,2{ }^{\circ} \mathrm{C}$ y una entalpía de fusión de 95, $3 \mathrm{~J} / \mathrm{g}$ (Serrano, 20 I2), sin embargo, ese valor no fue reflejado en el análisis.

La mezcla PEBD postconsumo reportó dos picos de temperatura, variando respecto a la temperatura de fusión teórica del PEBD que está entre $106-115^{\circ} \mathrm{C}$, lo cual implica que, al ser una mezcla heterogénea, tuvo variaciones en el encadenamiento de sus componentes y por ende en su temperatura.

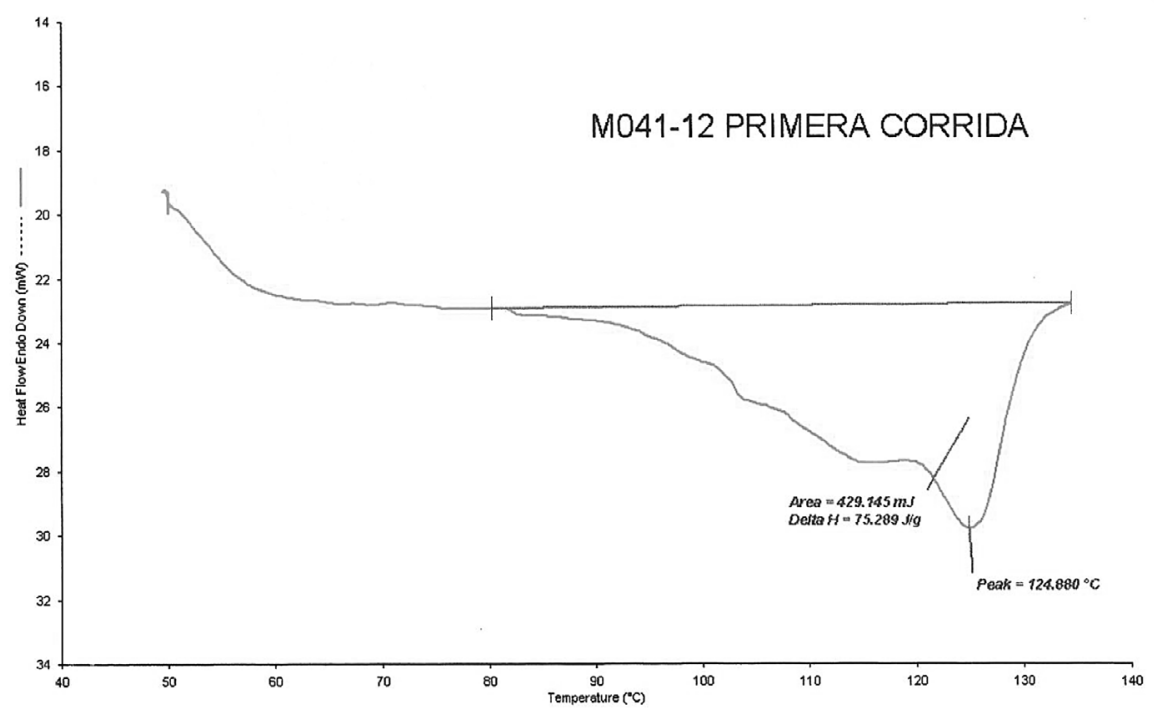

Figura 2. Gráfica de calorimetría diferencial de barrido del poliducto (DSC).

Cuadro 3. Resultados de la calorimetría diferencial de barrido (DSC) de las muestras.

\begin{tabular}{|c|c|c|}
\hline MUESTRA & $\begin{array}{c}\text { Temperatura de fusión más baja } \\
\left({ }^{\circ} \mathrm{C} \pm 0,00 \mathrm{I}\right)\end{array}$ & $\begin{array}{c}\text { Temperatura de fusión más alta } \\
\left({ }^{\circ} \mathrm{C} \pm 0,00 \mathrm{I}\right)\end{array}$ \\
\hline PEBD postconsumo & $111,80 \mathrm{I}$ & 126,218 \\
\hline PEAD-PP & $*$ & 130,929 \\
\hline PEAD-PEBD & 112,973 & 123,608 \\
\hline
\end{tabular}

Fuente: POLIUNA 2012. * Sin datos 
La mezcla de PEAD-PEBD también mostró dos picos; el inicial corresponde al PEBD con un valor I I 2,973 ${ }^{\circ} \mathrm{C}$ y otro valor de $123,608^{\circ} \mathrm{C}$, colocándose a una temperatura más baja del ámbito de fusión del PEAD, que, por la misma razón del caso anterior, pudo darse por modificaciones en sus propiedades de encadenamiento.

Para los resultados para TGA (Cuadro 4), se ha determinado la temperatura de inicio de la descomposición y la temperatura de finalización de todas las mezclas, así como la del poliducto, las cuales mostraron valores relativamente pequeños para las mezclas PEAD-PP y PEAD-PEBD, y un poco más altos para las mezcla del PEBD postconsumo y del poliducto. Esto se debe a que se produce una descomposición gradual de cada uno de sus componentes.

De forma general, en las mezclas de al menos dos componentes, la temperatura inicial de descomposición aumenta y el cambio de temperatura es mayor para los casos en que se considera un solo componente, por ejemplo, PEBD postconsumo y el poliducto, que ambos están constituidos solo de PEBD.

Las características estudiadas revelan que el poliducto de riego en estudio está elaborado con polietileno de baja densidad, al ser comparados con los parámetros mecánicos y térmicos existentes; y que la muestra que se ajusta a las propiedades de resistencia en elongación y flexión es la PEBD postconsumo, sin embargo, no cumple en su totalidad la resistencia al impacto necesaria para caracterizarla como materia prima en la elaboración de ducto de riego.

Las variaciones de las propiedades estudiadas en las mezclas se atribuyen a factores producidos en el proceso de extrusión, pues en algunos casos las láminas extruidas no alcanzaron su punto de fusión, dándose mezclas heterogéneas con temperaturas de operación menores a las necesarias para llegar a su fundición, esto permitió la maleabilidad de los polímeros, mas no su homogeneidad; lo que dejó como producto láminas heterogéneas y rugosas en su relieve, características que según Parma (20ll) son normales. Por lo tanto, se considera de gran importancia tener control permanente en el procesamiento de polímeros por extrusión, pues según Ingo Alig et al. (2010) "dado que la propiedad final del producto aparece en el estado sólido (cristalino o semicristalino) del polímero, existe una necesidad cada vez mayor de supervisar la línea durante el moldeo por inyección o extrusión de película plana. Lo mismo con el seguimiento de la estabilidad del proceso a lo largo de la línea de producción, con el fin de garantizar una calidad constante y fiabilidad de procesamiento".

\section{Conclusiones}

El material usado en el poliducto importado y utilizado como referencia en la elaboración de las otras muestras se caracteriza, por sus propiedades mecánicas y térmicas, como una resina de polietileno de baja densidad. Se llevó a cabo la obtención y caracterización térmica y mecánica de tres mezclas diferentes, a saber: PEBD postconsumo, PEAD-PP y PEAD-PEBD. De estas, la mejor formulación desarrollada a partir de materiales poliméricos postconsumo, para semejar o mejorar las características mecánicas del material del poliducto comercial, fue la mezcla de diferentes productos de PEBD postconsumo con $95 \%$ de confianza, cumpliendo con los requisitos de resistencia en elongación, resis-

Cuadro 4. Resultados del análisis termogravimétrico (TGA).

\begin{tabular}{|c|c|c|c|c|}
\hline MUESTRA & $\begin{array}{c}\text { Temp. inicial } \\
\left({ }^{\circ} \mathrm{C} \pm 0,0 \mathrm{I}\right)\end{array}$ & $\begin{array}{c}\text { Temp. final } \\
\left({ }^{\circ} \mathrm{C} \pm 0,0 \mathrm{I}\right)\end{array}$ & $\begin{array}{c}\text { Cambio de } \\
\text { Temperatura } \\
\left({ }^{\circ} \mathrm{C} \pm 0,0 \mathrm{I}\right)\end{array}$ & $\begin{array}{c}\text { Residuo } \\
(\% \pm 0,00 \mathrm{I})\end{array}$ \\
\hline PEBD postconsumo & 380,85 & 516,90 & 136,05 & $1,15 \mathrm{I}$ \\
\hline PEAD-PP & 428,59 & 512,88 & 84,29 & 2,995 \\
\hline PEAD-PEBD & 455,24 & 521,02 & 65,78 & 26 \\
\hline
\end{tabular}

Fuente: POLIUNA 2012. 
tencia en flexión, y necesitando la optimización de las características para resistencia al impacto modo IZOD. Además, las mezclas PEBD postconsumo y el poliducto tuvieron temperaturas de descomposición semejantes y mostraron cambios de temperatura de descomposición mayores que las mezclas PEAD-PP y PEAD-PEBD. Se logró determinar que la calidad de las láminas depende de la homogeneidad y miscibilidad de la mezcla y ella del seguimiento y control en el proceso de extrusión.

Dado el resultado de esta investigación, se considera necesaria la continuidad y profundización del tema, tomando en cuenta la mezcla óptima para la elaboración del poliducto, en tópicos como la observación del acoplamiento de las cadenas y la introducción de nanomateriales o bien aditivos que modifiquen sus propiedades mecánicas y térmicas con el fin de mejorarlas.

\section{Agradecimientos}

Este proyecto fue apoyado y financiado por el Tecnológico de Costa Rica, bajo la Actividad de Fortalecimiento "Formulación de un material para fabricar en Costa Rica poliducto de riego a partir de materiales poliméricos postconsumo generados en el desecho doméstico".

\section{Bibliografía}

Alig, I., Steinhoff, B. \& Lellinger, D. (20 I 0). Monitoring of polymer melt processing. Measurement Science Technology, 21 (6). doi: | 0. | 088/0957-0233/21/6/06200 |

Carraher, C. E. \& Seymour, R. B. (1995). Introducción a la química de los Polímeros. Barcelona: Editorial Reverté.

Harper, C. (1975). Handbook of Plastics and Elastomers. (Caps. I, 3 y 4). McGraw-Hill.

Kulshreshtha, A. K. \& Vasile, C. (2002). Handbook of Polymer Blends and Composites (Vols. 1-4). Smithers Rapra Technology. Recuperado de http://www.knovel.com/ web/portal/browse/display?_EXT_KNOVEL_DISPLAY_ bookid $=2219 \&$ VerticallD $=0$

Odian, G. (1991). Principles of Polymerization. 3 ed. New York: Wiley-Interscience.

Osswald, T. (20I I). Understanding polymer processing: processes and governing equation. Germany: Hanser Publications.

Parma, H. J. (20 I I). Incorporation of virgin and recycled thermoplastics in UPVC-based blends. University of Massachusetts Lowell. ProQuest Dissertations and Theses, 94. Recuperado de http://search.proquest.com/docview/873743863?accou ntid $=2765$ I

POLIUNA (2012). Informes de Ensayo No. 016-12 y 002-13. Laboratorio de Polímeros, Escuela de Química, Universidad Nacional de Costa Rica.

Programa Estado de la Nación. (2012). Decimoctavo Informe Estado de la Nación en Desarrollo Humano Sostenible. San José: Programa Estado de la Nación.

Serrano, G. (2012). Obtención y caracterización de mezclas de polipropileno y cera parafina para su aplicación en materiales de cambio de fase. Tesis de licenciatura en Ingeniería industrial. Departamento de Ciencia e Ingeniería de Materiales e Ingeniería Química. Escuela Politécnica Superior, Universidad Carlos III de Madrid. 